\title{
Simulations of solar cell absorption enhancement using resonant modes of a nanosphere array
}

\author{
Jonathan Grandidier*a, Michael G. Deceglie ${ }^{\mathrm{a}}$, Dennis M. Callahan ${ }^{\mathrm{a}}$, Harry A. Atwater ${ }^{\mathrm{a}}$ \\ ${ }^{a}$ Thomas J. Watson Laboratories of Applied Physics, California Institute of Technology, Pasadena, \\ CA 91125, USA; \\ b. Kavli Nanoscience Institute, California Institute of Technology, Pasadena, CA 91125, USA;
}

\begin{abstract}
We propose an approach for enhancing the absorption of thin-film amorphous silicon solar cells using periodic arrangements of resonant dielectric nanospheres deposited as a continuous film on top of a thin planar cell. We numerically demonstrate this enhancement using 3D full field finite difference time domain simulations and 3D finite element device physics simulations of a nanosphere array above a thin-film amorphous silicon solar cell structure featuring back reflector and anti-reflection coating. In addition, we use the full field finite difference time domain results as input to finite element device physics simulations to demonstrate that the enhanced absorption contributes to the current extracted from the device. We study the influence of a multi-sized array of spheres, compare spheres and domes and propose an analytical model based on the temporal coupled mode theory.
\end{abstract}

Keywords: Resonant Dielectric Structures, Solar cells, Nanospheres, Whispering gallery modes, Photovoltaics

\section{INTRODUCTION}

Dielectric nanospheres are promising structures for light trapping in planar thin-film solar cells. It has recently been shown that freely propagating sunlight can be diffractively coupled and transformed into several guided modes within an array of wavelength scale dielectric spheres. Incident optical power is then transferred to the thin-film absorber layer within the cell by leaky mode coupling. Guided whispering gallery modes in the spheres can be coupled into particular modes of the solar cell and significantly increase the fraction of incident light absorbed [1]. As a reference, we use a typical flat ultra-thin amorphous silicon (a-Si) solar cell and we study the influence of a hexagonally close packed (HCP) array of nanospheres on top of it. Spheres can be mono- or multi- sized, and we look at an array of wavelength scale dielectric spheres separated by smaller spheres. Using a multi-size array of spheres, additional mode excitation and therefore photocurrent improvement is expected. We numerically demonstrate this optical enhancement using 3D full field finite difference time domain (FDTD) simulations of a nanosphere array above a thin-film a-Si solar cell solar cell structure featuring back reflector and optimized anti-reflection coating. We also use the FDTD results as input to finite element device physics simulations to demonstrate that the enhanced absorption contributes to the current extracted from the device. We then compare the influence of dielectric spheres with nanodomes. All simulations presented here are performed with a commercial finite difference time domain simulator [2] and finite element device physics software [3]. Finally, we propose an analytical approach based on the temporal coupled mode theory.

\section{FLAT AMORPHOUS SILICON SOLAR CELL}

In order to have a control reference for our analysis, we first consider a typical flat a-Si solar cell structure as represented in Fig. 1a. It is composed of a silver (Ag) back reflector, a 130-nm aluminum-doped zinc oxide (AZO) layer, a 100-nm a-Si [4] layer and an 80-nm thick indium tin oxide (ITO) layer. The AZO layer prevents diffusion of silver in a-Si. The ITO layer is used as a transparent front contact and acts as an optimized anti-reflection coating. We use 2D FDTD simulations to calculate the absorption in the a-Si layer. To simulate solar illumination, we use a polarized broad band plane wave source. We verify that the same absorption is calculated in both transverse magnetic and transverse electric polarizations. We record the electromagnetic fields for 300 equally spaced frequencies between $\lambda=300 \mathrm{~nm}$ and $\lambda=840 \mathrm{~nm}$ corresponding to the above band gap absorption in a-Si. In the active region, the power absorbed per unit volume can be calculated from the divergence of the Poynting vector and is equivalent to [5]

Physics, Simulation, and Photonic Engineering of Photovoltaic Devices,

Edited by Alexandre Freundlich, Jean-Francois F. Guillemoles, Proc. of SPIE Vol. 8256, 825603

(C) 2012 SPIE · CCC code: 0277-786X/12/\$18 - doi: 10.1117/12.909677

Proc. of SPIE Vol. 8256 825603-1 


$$
P_{a b s}^{a-S i}(\lambda)=\frac{1}{2} \omega \varepsilon^{\prime \prime}|E(\lambda)|^{2}
$$

where $|E(\lambda)|^{2}$ is the magnitude of the electric field, $\omega=2 \pi c / \lambda$ is the angular frequency of the light where $c$ is the speed of light in vacuum, and $\varepsilon^{\prime \prime}$ is the imaginary part of the dielectric permittivity.

In the FDTD simulation, the material is defined in every simulation grid point. From Eq. 1, it is clear that the power absorbed is directly related to the intensity of the electric field. It is therefore of great importance to increase the electric field intensity in the active region in order to increase its absorption. The only useful absorption is the one that occurs in the active layer. Absorption in other layers is parasitic. Since the a-Si absorbing layer is very thin, we assume that for each photon absorbed in the a-Si, one electron - hole pair is collected. This corresponds to unity internal quantum efficiency (IQE) in the a-Si layer. With $P_{\text {incident }}(\lambda)$ being the incident power per unit area, the absorption in one of the materials constituting the solar cell is calculated by monitoring the power that enters $P_{\text {in }}$ and exists $P_{\text {out }}$ the considered material

$$
A B S(\lambda)=\frac{P_{\text {in }}(\lambda)-P_{\text {out }}(\lambda)}{P_{\text {incident }}(\lambda)}
$$

The solar cell is illuminated under the AM1.5G solar spectrum [6]. Using the absorption in the a-Si layer, we calculate the absorbed photocurrent

$$
J_{p h}(\lambda)=A B S^{a-S i}(\lambda) * J_{\text {solar }}(\lambda)
$$

where

$$
J_{\text {solar }}(\lambda)=N_{0}(\lambda) * e^{-}
$$

is the equivalent spectral current density for the AM1.5G reference spectrum. $e^{-}$is the electron charge,

$$
N_{0}(\lambda)=\frac{P_{\text {solar }}(\lambda)}{E_{p h}(\lambda)}
$$

is the incident photon flux. $P_{\text {solar }}$ is the equivalent spectral power density for the AM1.5G reference spectrum and $\operatorname{Eph}(\lambda)=h c / \lambda$ is the photon energy where $h$ is Planck's constant and $c$ is the speed of light in vacuum.
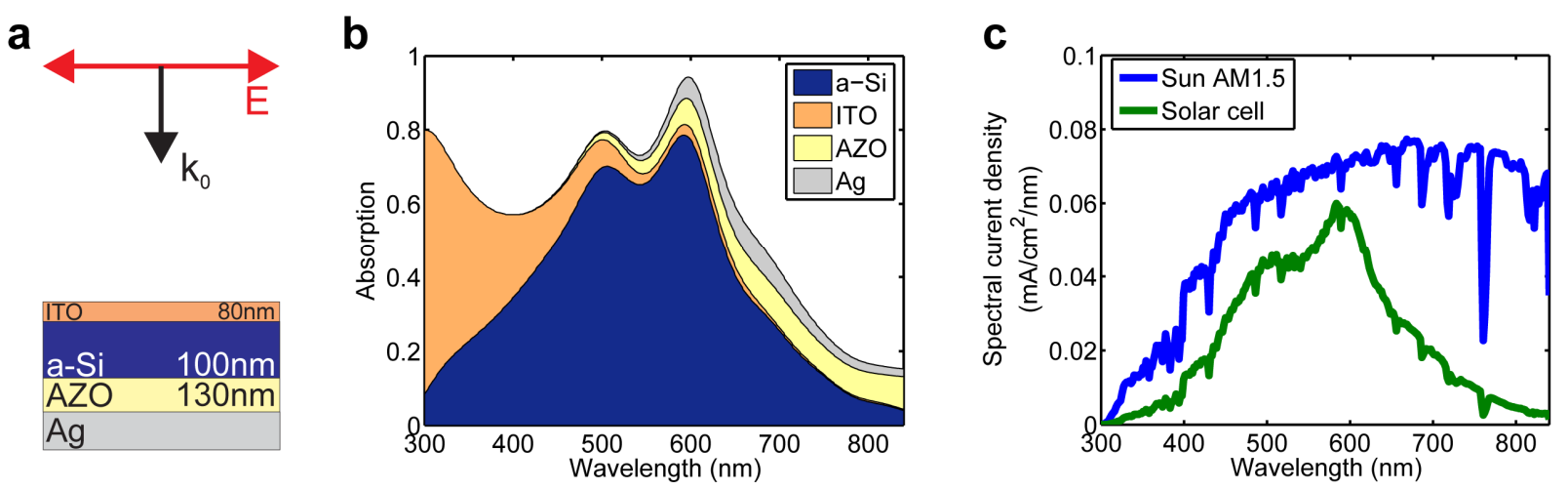

Figure 1. (a) Representation of the flat a-Si structure. (b) Absorption calculated in the different parts of the solar cell as a function of the illumination wavelength. For the a-Si layer, this corresponds to the external quantum efficiency assuming that each photon absorbed in the semiconductor creates one electron - hole pair. (c) Spectral absorbed photocurrent density $\left(J_{p h}\right)$ of the solar cell illuminated by the AM1.5G solar spectrum. $J_{\text {solar }}$ is also represented.

Figure $1 \mathrm{~b}$ shows the calculated absorption in each layer of the considered solar cell. In the blue part of the spectrum, most of the photons are lost in the first $80 \mathrm{~nm}$ of the ITO layer. For the a-Si layer, the absorption generates photocurrent. In order to calculate the total generated absorbed photocurrent, $J_{p h}$, under the AM1.5G solar spectrum at 
normal incidence, the absorption in the a-Si is weighted by the solar spectrum (Fig. 1c). We calculate $J_{p h}=12.47 \mathrm{~mA} / \mathrm{cm}^{2}$. Note that in the considered spectral range, this value corresponds to $42 \%$ of the maximum attainable value of 29.90 $\mathrm{mA} / \mathrm{cm}^{2}$.

In order to understand the link between optical and electrical performance [7, 8], we use the results of the FDTD modeling as input into 3D finite-element device physics simulations. The generation rate at each point on the FDTD simulation grid is calculated from

$$
G_{\text {opt }}(\lambda)=\frac{\varepsilon^{\prime \prime}|E(\lambda)|^{2}}{2 \hbar}
$$

which is then weighted by the solar spectrum. This 3D generation profile is then interpolated onto the finite-element device physics simulation mesh and the electrical current extracted from the device is calculated for changing applied voltage, by numerically solving the electrostatic and carrier transport equations in the a-Si layer [9].

We consider an n-i-p device. The top $10 \mathrm{~nm}$ of the a-Si is doped p-type and the bottom $10 \mathrm{~nm}$ are doped n-type, both at active dopant concentrations of $3 \times 10^{19} \mathrm{~cm}^{-3}$, the rest is intrinsic. All layers are assumed to have a mobility band gap of $1.78 \mathrm{eV}$, the trap density in the intrinsic region is taken as $2 \times 1017 \mathrm{~cm}^{-3}$, and Ohmic contacts are assumed. All other electrical parameters are taken from Schropp and Zeman [10]. From these calculations we extract values for the short circuit current $J_{s c}$ open circuit voltage $V_{o c}$ and fill factor $F F$. The efficiency $\eta$ is then calculated according to [11] from

$$
\eta=\frac{F F * J_{s c} * V_{o c}}{P_{i n}} .
$$

We calculate an overall conversion efficiency of $7.61 \%$, with $J_{s c}$ of $9.27 \mathrm{~mA} / \mathrm{cm}^{2}, V_{o c}$ of $0.990 \mathrm{~V}$, and $F F$ of 0.830 . Note that $J_{s c}$ is lower than $J_{p h}$ due to imperfect charge carrier collection within the device.

\section{LIGHT TRAPPING USING RESONANT SPHERES}

\subsection{Description of the concept}

Our approach here is to consider an array of hexagonally close packed resonant spheres on top of the solar cell described in the previous section. We demonstrated previously the interest of $\mathrm{SiO}_{2}$ spheres directly placed on top of a-Si [1] and a gallium arsenide (GaAs) [12] solar cells. We extend this concept and compare the influence of different configurations presented in the next sections.
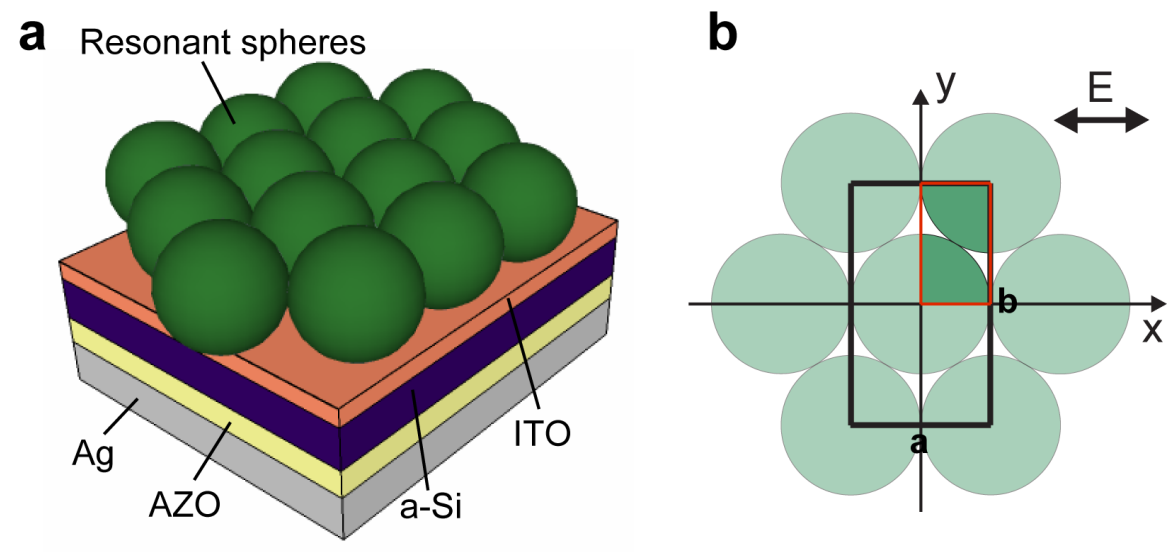

Figure 2. (a) Schematic of the solar cell with resonant spheres. (b) Top view of the periodic arrangement of spheres with their lattice constant $a$. The black rectangle of dimension $a, b$ indicates the unit cell and the red rectangle indicates the region that is effectively used for numerical simulations.

Figure 2a describes the base used for the simulations. Resonant spheres are deposited on top of the a-Si solar cell. Here, the spheres are hexagonally close packed and the lattice constant $a$ is equal to the diameter of the spheres $D$. Because the system is periodic, we only simulate one period of the structure corresponding to the rectangle of dimension $a, b$ shown in Fig. 2b. We use periodic boundary conditions in our 3D FDTD simulations to account for the infinite 
periodicity of the structure as well as for the broadband normal incident plane wave source. In this HCP configuration, the dimensions of the unit cell are $a$ and $b=a \sqrt{3}$. The symmetry of the unit cell and the orientation of the electromagnetic field allow us to simulate one quadrant of the unit cell as indicated in Fig. 2b, thus significantly reducing the time and memory requirements for the simulation.

\subsection{Electrical performance}

We use the device physics model, described in the previous section for the case of the flat a-Si solar cell, to simulate the electrical performance of cell enhanced with $600 \mathrm{~nm}$ diameter hexagonally close packed resonant $\mathrm{SiO}_{2}$ spheres on top of it. We find that the absorbed photocurrent, $J_{p h}$, is enhanced with respect to the flat device to a total $14.05 \mathrm{~mA} / \mathrm{cm}^{2}$, resulting in an increased $J_{s c}$ of $10.37 \mathrm{~mA} / \mathrm{cm}^{2}$, compared to a $J_{s c}$ of $9.27 \mathrm{~mA} / \mathrm{cm}^{2}$ in the flat case. As we expect, $V_{o c}$ is very close to the case without spheres and equals $0.993 \mathrm{~V}$. $F F$ is 0.831 . The result is that the calculated efficiency is improved to $8.55 \%$, a relative improvement of $12.3 \%$ compared to the flat cell.
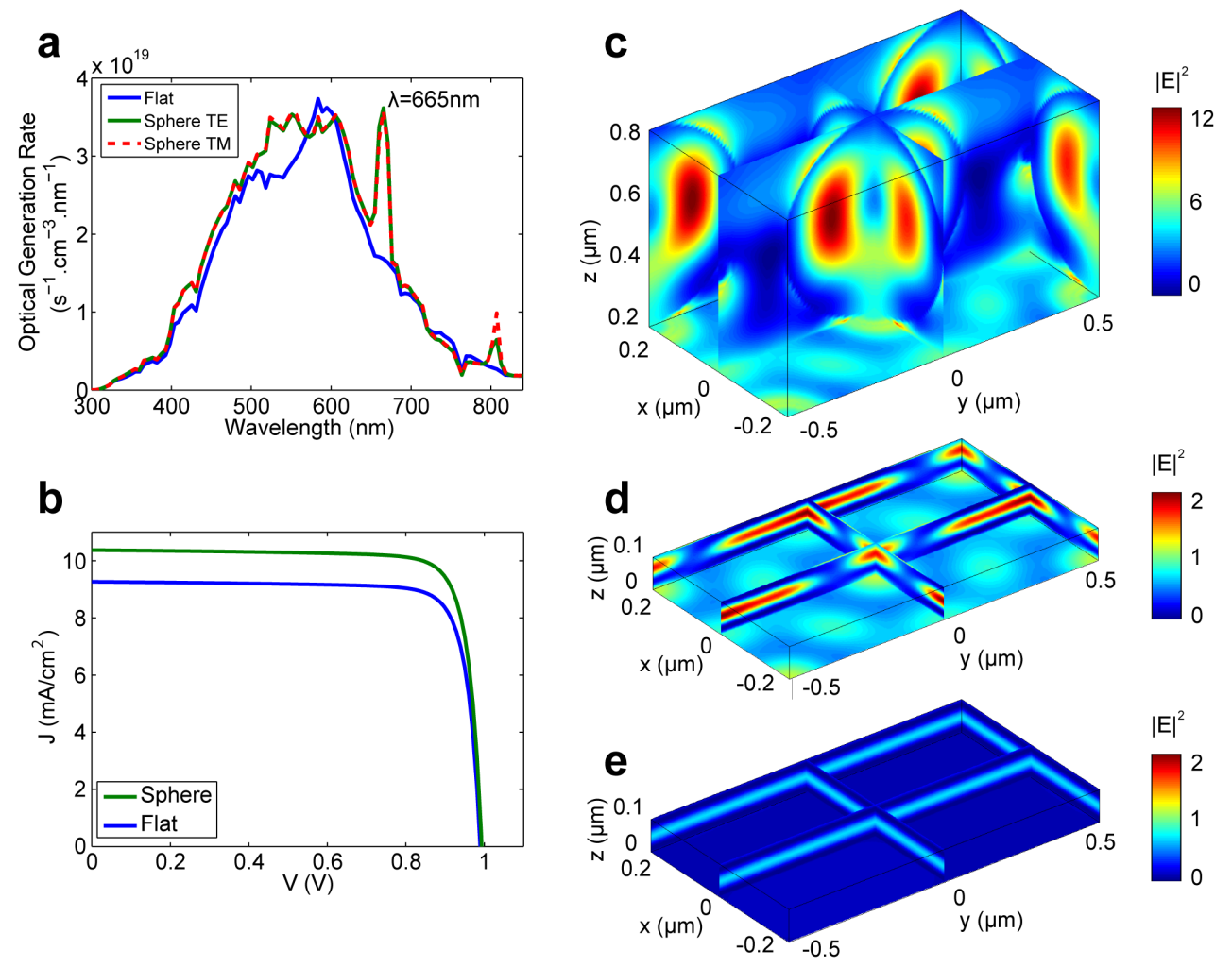

Figure 3. (a) Optical generation rate calculated in the amorphous silicon with and without the presence of dielectric nanospheres. (b) Calculated current-voltage (JV) curve for the case with and without spheres. (c,d) Averaged TE and TM electric field intensity in the region where the spheres are present and in the a-Si region respectively at the resonant frequency $\lambda=665 \mathrm{~nm}$. (e) Electric field intensity in the a-Si region for the flat case at $\lambda=665 \mathrm{~nm}$.

The fact that the improvement in $J_{p h}$ results in a corresponding improvement in $J_{p h}$ indicates that the carriers generated by the resonant absorption are electrically collected within the device. As is apparent in Fig. 3d, much of the enhancement occurs in the intrinsic region, thus avoiding absorption in the doped regions, which have higher trap densities, and thus degraded carrier lifetimes [10]. We also perform these coupled optical and electrical simulations at specific wavelengths and verify that the carriers generated at the resonant wavelengths contribute to the overall $J_{s c}$. At the resonant wavelength of $665 \mathrm{~nm}$ (Fig. 3), we find an external quantum efficiency of 0.66 , improved from only 0.32 in the flat case, resulting from the increased absorption of solar radiation in the a-Si, and the subsequent collection of the generated carriers.

We observe that even though the total optical generation rate is the same for TE (Transverse Electric) and TM (Transverse Magnetic) polarizations (Fig. 3c and d), the field profile is different (not shown here). For the device 
physics, we consider the averaged value of TE and TM. Even though it is more precise, recording the electric field in each simulation grid point during FDTD calculations is very memory consuming. Therefore, we consider 100 points equally spaced frequencies between $\lambda=300 \mathrm{~nm}$ and $\lambda=840 \mathrm{~nm}$. For the following sections, we calculate the absorbed photocurrent density using the absorption (Eq. 2) in TE polarization and consider 300 points.

\section{MULTI-SIZE SPHERE ARRAY}

Here we consider a multi-sized sphere configuration. This could result in adding a larger number of resonances and therefore increase further the current density of the solar cell.

\subsection{Multi-sized sphere arrangement}

Our simulation approach is to start from the same hexagonally close packed sphere arrangement as shown in Fig. $2 b$ and to insert smaller spheres that fit between the ITO top layer of the solar cell and the bigger spheres [13].
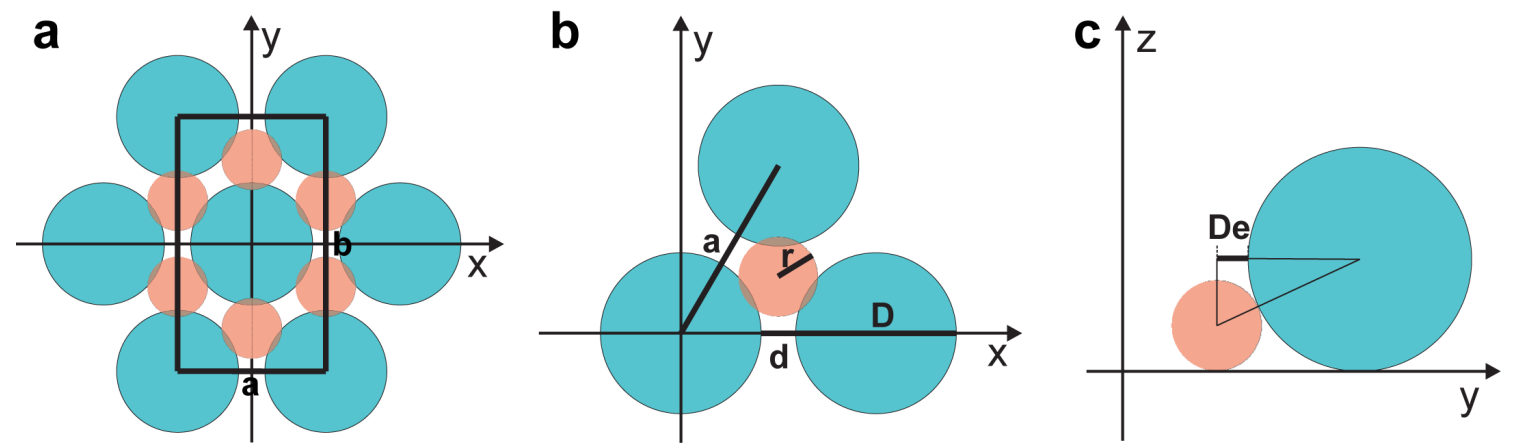

Figure 4. (a,b) Top view of the periodic arrangement of multi-sized spheres with their lattice constant $a$. The black rectangle of dimension $a, b$ indicates the unit cell used for numerical simulations. (c) Side view of 2 spheres of different sizes used for the simulations.

We represent in Fig. 4 the multi-sized sphere arrangement used for the numerical simulations. Two sizes of spheres are used. For the bigger spheres, their arrangement is the same as the one shown in Fig. $2 \mathrm{~b}$ with a spacing $d$ between the spheres. Therefore, the lattice constant is $a=D+d$. The dimensions of the unit cell are $a$ and $b=(D+d) \sqrt{3}$. The smaller spheres are placed in the space formed by three nearby bigger spheres as shown in Fig. 4b. One smaller sphere fits in such a way that it is in contact with the bigger spheres as well as with the layer under it. In that way, all the spheres are close packed and the whole multi-sized sphere structure maintains itself on top of the solar cell. To place the smaller spheres in the structure, we define their position relatively to the bigger spheres as shown in Fig. 4 as $D e=\frac{\sqrt{3}}{3}(D+d)-\frac{D}{2}$ and their radius equals

$$
r=\frac{D}{8}+\frac{D e}{2}+\frac{D e^{2}}{2 D}
$$

\subsection{Simulations of a multi-sized sphere structure}

Using the a-Si solar cell as above, we compute a set of FDTD simulations with a multi-sized sphere array on top of it. For this purpose, we use $\mathrm{SiO}_{2}$ for all the spheres. We keep the bigger sphere diameter constant to $D=600 \mathrm{~nm}$ and we vary the spacing $d$ between 0 and $220 \mathrm{~nm}$ by steps of $20 \mathrm{~nm}$. When $d=0$, the bigger spheres are hexagonally close packed and the smaller spheres have a radius of $r=100 \mathrm{~nm}$. When $d$ increases, $r$ increases as described in Eq. 8. The calculated absorbed photocurrent density is represented in Fig. 5a as a function of d. To evaluate the influence of the multi-sized sphere structure, we also compute the absorbed photocurrent density for a case without the smaller spheres. For the multi-sized sphere structure, the highest absorbed photocurrent density is obtained for $d=120 \mathrm{~nm}$ and equals $J_{S C}=14.50$ $\mathrm{mA} / \mathrm{cm}^{2}$ which corresponds to an enhancement of about $16 \%$ compared to the case without the sphere array. In this case, the smaller spheres have a radius of $r=144 \mathrm{~nm}$. This value for the absorbed photocurrent density is very similar to the 
calculated absorbed photocurrent density for $d=60 \mathrm{~nm}$ without the smaller spheres. As a comparison, we represent in Fig. $5 \mathrm{~b}$ the spectral absorbed photocurrent density for $d=120 \mathrm{~nm}$. The multi-sized sphere configuration gives a slightly higher absorbed photocurrent density than the mono-sized configuration, but the spectral enhancement peaks occur at similar wavelengths.

a

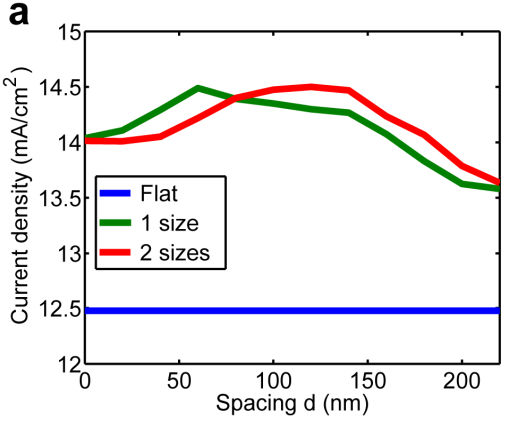

b

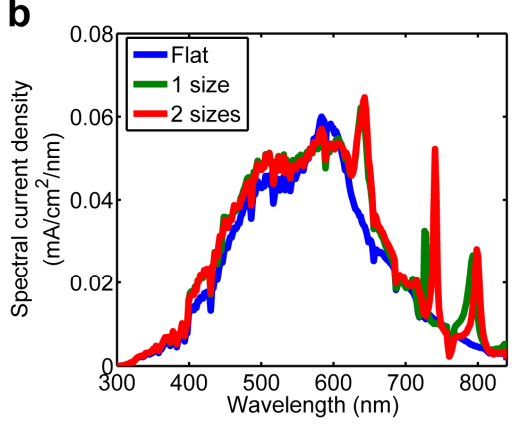

C

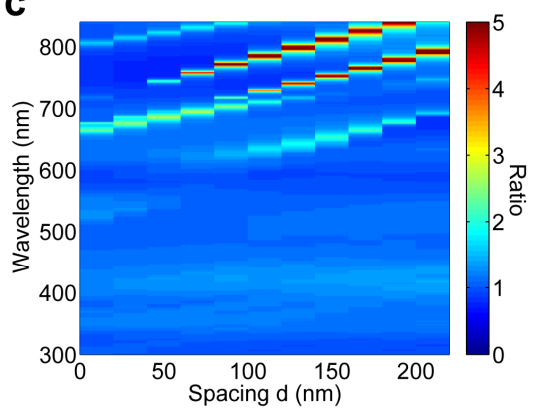

Figure 5. (a) Absorbed photocurrent density of a solar cell with a multi-sized and a mono-sized sphere structure as a function of the distance between the bigger spheres. (b) Corresponding spectral absorbed photocurrent density for the optimal multi-sized configuration where $d=120 \mathrm{~nm}$. (c) Ratio between the spectral absorbed photocurrent density of an a-Si silicon solar cell with multi-sized spheres over the spectral absorbed photocurrent density of a solar cell without spheres.

In Fig. 5c, we illustrate the ratio between the spectral absorbed photocurrent density of a solar cell with multi-sized sphere configuration over the spectral absorbed photocurrent of a solar cell without spheres. In all cases, strong enhancement occurs and indicates the optical dispersion of the modes associated with the multi-sized sphere array. This analysis suggests that the enhancement is mainly due to a coupling of the free space light into the periodic arranged structure. The sphere array on top of the solar cell can be seen as a low loss photonic crystal [14] and the energy present in each excited modes leaks into the solar cell underneath due to the index mismatch between the sphere array and the solar cell.

\section{COMPARISON BETWEEN SPHERES AND DOMES}

In this section, we compare the absorbed photocurrent density obtained for a case with $\mathrm{SiO}_{2}$ spheres and a case with $\mathrm{SiO}_{2}$ domes. Nanodome structures have already shown their interest for photovoltaics applications [15]. For this purpose, we consider the solar cell structure as represented on Fig. 6. It is composed of a silver back reflector, a 200nm flat a-Si layer and a silicon nitride $\left(\mathrm{Si}_{3} \mathrm{~N}_{4}\right)$ layer of thickness $t$. Silicon nitride is a commonly used anti-reflection coating for silicon solar cells [16]. We perform FDTD simulations and calculate the absorbed photocurrent density for three different cases. In the first one, we vary the silicon nitride thickness between $20 \mathrm{~nm}$ and $300 \mathrm{~nm}$ (Fig. 6a). In the second one, sphere domes are considered (Fig. 6b). When the silicon nitride becomes 300nm thick, $\mathrm{SiO}_{2}$ hemispheres remain on it. In the third one, we simulate partially embedded $600 \mathrm{~nm}$ spheres (Fig. 6c). As opposed to the previous sections, the spheres or domes are arranged in a square lattice.

a

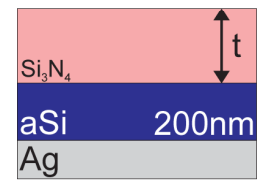

b

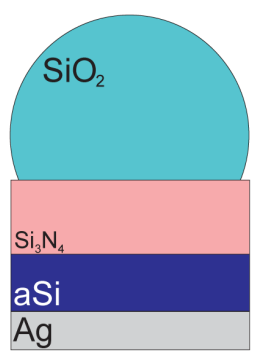

C

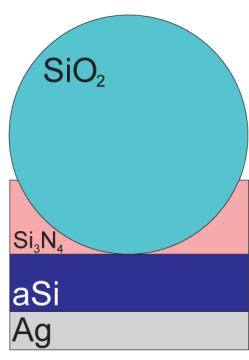

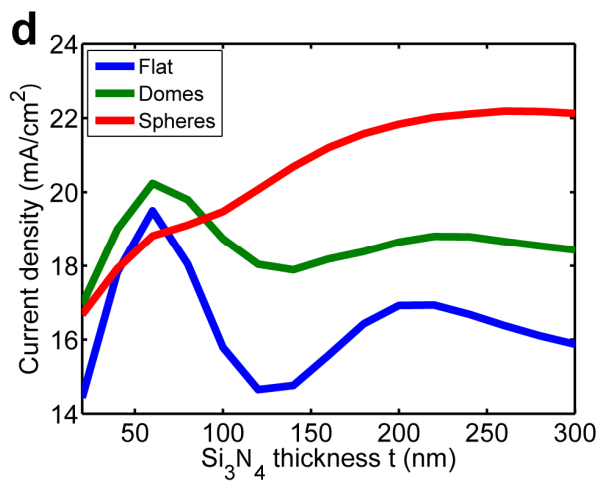

Figure 6. (a) Flat solar cell structure with a silicon nitride antireflection coating. (b) Equivalent case with nanodomes and (c) nanospheres. (d) Calculated absorbed photocurrent density $\left(J_{p h}\right)$ for (a), (b) and (c). 
The result of this analysis is presented on Fig. 6d. For the flat case, the highest absorbed photocurrent density of $19.5 \mathrm{~mA} / \mathrm{cm}^{2}$ is obtained for a $60 \mathrm{~nm}$ thick silicon nitride layer. For the case with $\mathrm{SiO} 2$ domes, the highest absorbed photocurrent density is also obtained for a $60 \mathrm{~nm}$ thick silicon nitride layer. The recorded value of $20.24 \mathrm{~mA} / \mathrm{cm}^{2}$ is slightly higher. In those two first cases, a $60 \mathrm{~nm}$ thick silicon nitride layer appears to be the optimal anti-reflection coating at normal incidence. Nanodomes slightly increase the absorbed photocurrent density. However, the trend of the curve is similar to the flat case. This suggests that nanodomes act as an additional anti-reflection coating. For the case with spheres partially embedded, we calculate an absorbed photocurrent density of $22.19 \mathrm{~mA} / \mathrm{cm}^{2}$. This value is obtained for a $\mathrm{t}=260 \mathrm{~nm}$. This is more than $10 \%$ higher than the optimal case with nanodomes. This third case demonstrates the interest of combined dielectric spheres and anti-reflection coatings. The strong localized modes supported and excited within the spheres [17] have a direct influence for increasing the absorption in the active layer [12].

\section{TEMPORAL COUPLED MODE THEORY}

There are many parameters which need to be optimized for this particular light trapping concept, including the refractive index, diameter and lattice constant of the spheres, the separation from the solar cell, the refractive index of the spacer layer, the refractive index of the solar cell, etc. Scanning through all of these parameters with FDTD is computationally expensive and time consuming. Thus, we seek alternative analytic or semi-analytic ways to model the system. One such method is the use of temporal coupled mode theory $[18,19]$, which breaks down a system of interest into simple components such as waveguides and cavities. In Fig. 7a, we show how a solar cell with a microsphere array can be modeled using this method. Here we treat free space as a waveguide where light is incident from, the sphere array as a cavity with resonance $\omega_{0}$ which captures and stores the input energy for a finite time, and the solar cell as a semiinfinite waveguide to which light can leak from the sphere array. The lifetime for light leakage from the array to free space is given by $\tau_{1}$ and for leakage into the underlying solar cell $\tau_{2}$.
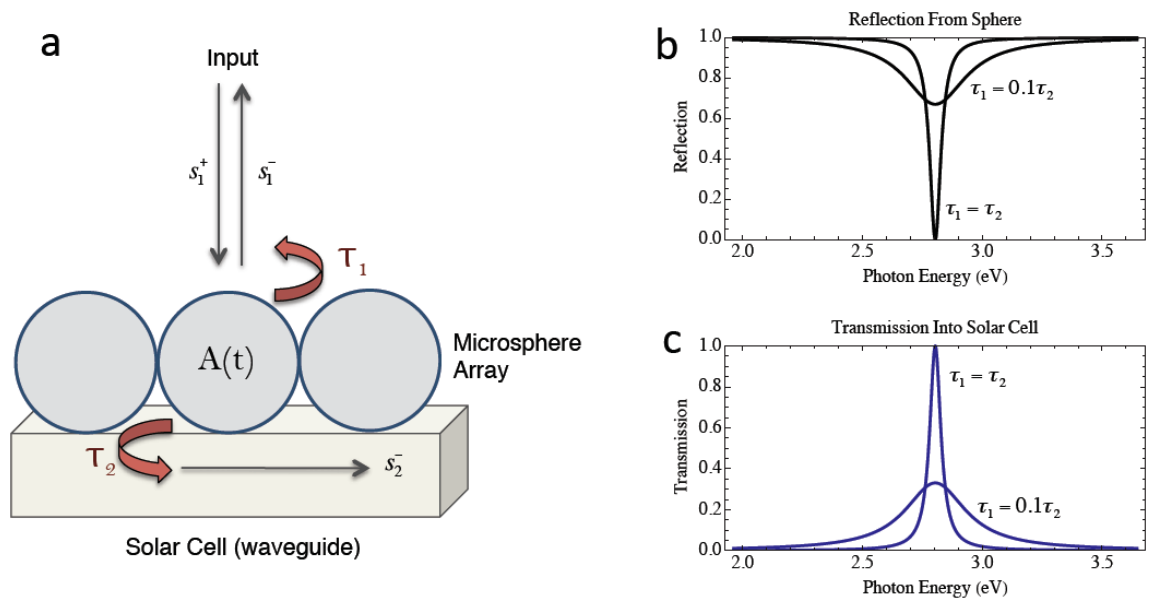

Figure 7. (a) Schematic representation of the simplified system used for modeling with temporal coupled mode theory. (b), (c) reflection and transmission spectrum based on the model, respectively.

If we define $|\mathrm{A}|^{2}$ as the energy stored within the microsphere array and $\left|\mathrm{s}^{ \pm}\right|$as the incoming/outgoing power in the waveguides, we can write a differential equation for the time dependence of the field amplitude within the resonator

$$
\frac{d A}{d t}=i \omega A=i \omega_{o} A-\frac{A}{\tau_{1}}-\frac{A}{\tau_{2}}+\sqrt{\frac{2}{\tau_{1}}} s_{1}^{+}
$$

with

$$
s_{1}^{-}=-s_{1}^{+}+\sqrt{\frac{2}{\tau_{1}}} A
$$

For more details, the reader is directed to the above references. We can solve these equations for the input and output powers to obtain expressions for the reflection and transmission spectra 


$$
\begin{gathered}
R(\omega)=\frac{\left|s_{1}^{-}\right|^{2}}{\left|s_{1}^{+}\right|^{2}}=\frac{\left(\omega-\omega_{o}\right)^{2}+\left(\frac{1}{\tau_{1}}-\frac{1}{\tau_{2}}\right)^{2}}{\left(\omega-\omega_{o}\right)^{2}+\left(\frac{1}{\tau_{1}}+\frac{1}{\tau_{2}}\right)^{2}} \\
T(\omega)=\frac{\left|s_{2}^{-}\right|^{2}}{\left|s_{1}^{+}\right|^{2}}=\frac{\frac{4}{\tau_{1} \tau_{2}}}{\left(\omega-\omega_{o}\right)^{2}+\left(\frac{1}{\tau_{1}}+\frac{1}{\tau_{2}}\right)^{2}}
\end{gathered}
$$

It is clear from these equations that, within this model, the reflection will go zero when the values for $\tau_{1}$ and $\tau_{2}$ are equal. We plot these spectra in Fig. 7b, c to further illustrate this.
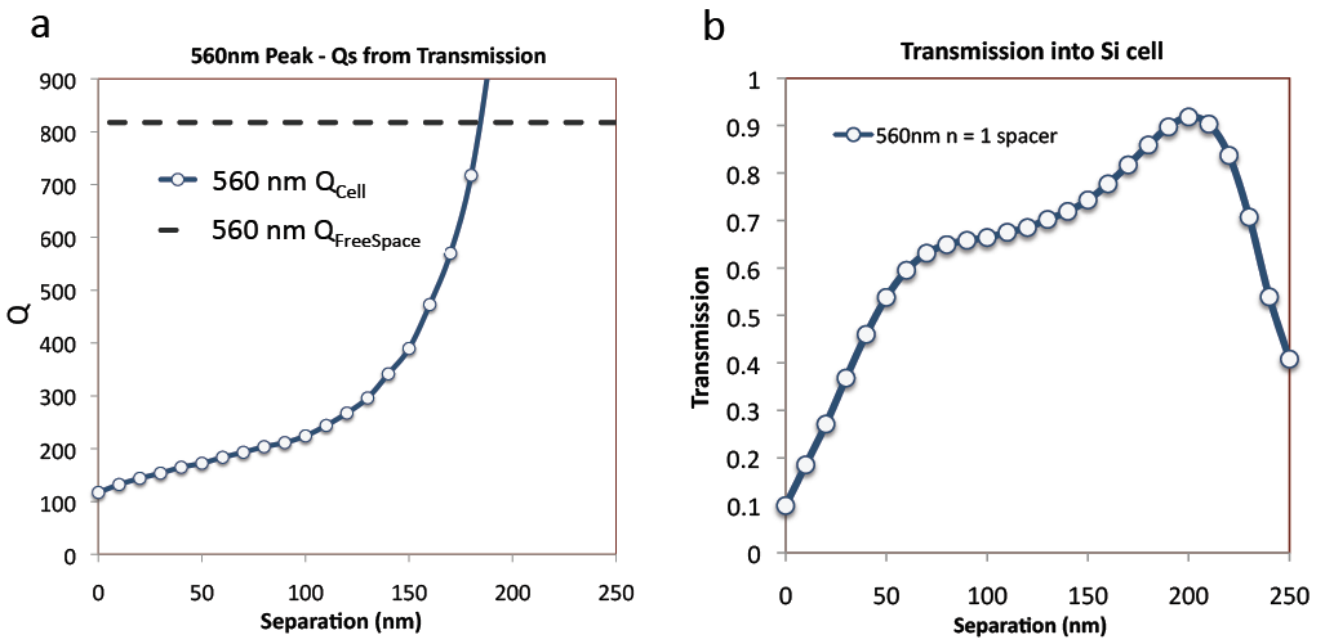

Figure 8. (a) Quality factors as a function of sphere array-solar cell separation. (b), transmission at $560 \mathrm{~nm}$ as a function of sphere array-solar cell separation.

We now take a simple system to apply this model to and compare with the predictions. We take an array of $500 \mathrm{~nm}$ close packed $\mathrm{SiO}_{2}$ spheres and separate them from a semi-inifinite cristalline silicon layer by various spacings of air. This configuration has numberous resonances, but we will focus on only one resonance at $560 \mathrm{~nm}$. By varying the separation, we are able to tune the value of $\tau_{2}$. We assume the value of $\tau_{1}$ is constant with separation. This is a good assumption as long as the modes of the array are weakly perturbed by the waveguide [18]. We can calculate the value of $\tau_{1}$ from the linewidth of the transmission spectrum for an array of spheres in free space. We then can calculate the value of $\tau_{2}$ from the quality factor from the linewidth of the transmission spectrum at each separation using the equation

$$
\frac{1}{Q}=\frac{1}{Q_{\text {FreeSpace }}}+\frac{1}{Q_{\text {Cell }}}
$$

where $\mathrm{Q}_{\text {FreeSpace }}=\omega \tau_{1}$ and $\mathrm{Q}_{\text {Cell }}=\omega \tau_{2}$. In Fig. 8a we plot the values of $\mathrm{Q}_{\text {cell }}$ for different sphere array-solar cell separations as well as the assumed constant value for $\mathrm{Q}_{\text {FreeSpace }}$. We see that $\mathrm{Q}_{\text {cell }}$ exponentially decreases as the sphere array is brought closer to the Si layer. The intersection of these two curves is where the condition for maximum transmission is met according to the model. To check the model, Fig. $8 \mathrm{~b}$ shows the FDTD calculated values for transmission at the resonant wavelength of $560 \mathrm{~nm}$.

A maximum in transmission occurs close to the expected position predicted by the theory at $\sim 200 \mathrm{~nm}$ separation. Further work is needed to refine this theory for all possible configurations, but these initial results suggest that temporal coupled mode theory could be used to model this system and to obtain informative predictions. 


\section{CONCLUSION}

We studied several configurations to enhance the light absorption within an ultra-thin a-Si solar cell by using a periodic nanosphere array on top of it. Multi-sized sphere arrays were studied and showed a similar enhancement as mono-sized spheres. By coupling optical and electrical simulation, we are able to verify that the enhanced absorption results in charge carriers that contribute to the electrical current generated by the cell. Finally, we demonstrated the advantages of dielectric resonant spheres over an equivalent nanodome structure and proposed an analytical model based on the temporal coupled mode theory.

\section{ACKNOWLEDGEMENT}

The authors wish to thank Raymond A. Weitekamp and Daniel B. Turner-Evans for useful technical input regarding simulations. The authors acknowledge support from the DOE "Light-Material Interactions in Energy Conversion" Energy Frontier Research Center under grant DE-SC0001293.

\section{REFERENCES}

[1] J. Grandidier, D. M. Callahan, J. N. Munday et al., "Light Absorption Enhancement in Thin-Film Solar Cells Using Whispering Gallery Modes in Dielectric Nanospheres," Advanced Materials, 23(10), 1272-1276 (2011).

[2] http://www.lumerical.com.

[3] http://www.synopsys.com/Tools/TCAD/Pages/default.aspx.

[4] http://www.sopra-sa.com.

[5] J. D. Jackson, [Classical Electrodynamics] Wiley, New York, 3rd Edition (1999).

[6] http://rredc.nrel.gov/solar/spectra/am1.5/.

[7] M. D. Kelzenberg, D. B. Turner-Evans, M. C. Putnam et al., "High-performance Si microwire photovoltaics," Energy \& Environmental Science, 4(3), 866-871 (2011).

[8] X. Li, N. P. Hylton, V. Giannini et al., "Bridging electromagnetic and carrier transport calculations for threedimensional modelling of plasmonic solar cells," Opt. Express, 19(S4), A888-A896 (2011).

[9] S. M. Sze, and K. K. Ng, [Physics of semiconductor devices] Wiley, Hoboken NJ, 3rd edition (2003).

[10] R. E. I. Schropp, and M. Zeman, [Amorphous and Microcrystalline Silicon Solar Cells: Modeling, Materials, and Device Technology] Kluwer Academic, Norwell MA (1998).

[11] J. Nelson, [The Physics of Solar Cells] Imperial College Press, London (2009).

[12] J. Grandidier, D. M. Callahan, J. N. Munday et al., "Gallium Arsenide Solar Cell Absorption Enhancement Using Whispering Gallery Modes of Dielectric Nanospheres," IEEE Journal of Photovoltaics, DOI: 10.1109/JPHOTOV.2011.2180512 (2012).

[13] V. Kitaev, and G. A. Ozin, "Self-Assembled Surface Patterns of Binary Colloidal Crystals," Advanced Materials, 15(1), 75-78 (2003).

[14] P. Bermel, C. Luo, L. Zeng et al., "Improving thin-film crystalline silicon solar cell efficiencies with photonic crystals," Opt. Express, 15(25), 16986-17000 (2007).

[15] J. Zhu, C.-M. Hsu, Z. Yu et al., "Nanodome Solar Cells with Efficient Light Management and Self-Cleaning," Nano Letters, 10(6), 1979-1984 (2009).

[16] B. Kumar, T. Baskara Pandian, E. Sreekiran et al., "Benefit of dual layer silicon nitride anti-reflection coating." Proc. PVSC, 1205-1208 (2005).

[17] A. N. Oraevsky, "Whispering-gallery waves," Quantum Electronics, 32(5), 377-400 (2002).

[18] J. D. Joannopoulos, S. G. Johnson, J. N. Winn et al., [Photonic Crystals: Molding the Flow of Light] Princeton University Press, Princeton NJ, 2nd edition (2008).

[19] Z. Yu, A. Raman, and S. Fan, "Fundamental limit of nanophotonic light trapping in solar cells," PNAS. Proc. Natl Acad. Sci. 107, 17491-17496 (2010). 